\title{
Random copolymers at a selective interface: many chains with excluded volume interactions
}

\author{
Gongwen Peng ${ }^{\dagger, \S}$, Jens-Uwe Sommer ${ }^{\dagger}$ and Alexander Blumen ${ }^{\dagger}$ \\ †) Theoretische Polymerphysik, Universität Freiburg, Rheinstr.12, D-79104 Freiburg, Germany \\ $\S)$ Department of Physics and Astronomy, Bowling Green State University, Bowling Green, OH 43403
}

October 4, 2018

\begin{abstract}
We investigate numerically, using the bond-fluctuation model, the adsorption of many random $\mathrm{AB}$-copolymers with excluded volume interactions at the interface between two solvents. We find two regimes, controlled by the total number of polymers. In the first (dilute) regime, the copolymers near the interface extend parallel to it, while in the second regime they extend perpendicular to it. The density at the interface and the density in the bulk depend differently on the total number of copolymers: In the first regime the density at the interface increases more rapidly then in the bulk, whereas the opposite is true in the second regime.
\end{abstract}

PACS numbers: $61.25 . \mathrm{Hq}, 83.70 . \mathrm{Hq}$ 


\section{Introduction}

Recently copolymers at selective interfaces have received much attention [16]. Consider as an example a block copolymer consisting of a hydrophilic and a hydrophobic part and situated next to an oil-water interface. The difference in the monomers' selectivity (which we denote by $k T \chi$ ) favors the localization of the copolymer at the interface, with each block in its favorable solvent. However, for random copolymers frustrated situations may arise, since the chain's connectivity forces some monomers to stay in their unfavorable solvent. Garel et al [3] have studied the localization transition of an ideal random chain at a selective interface. In previous publications [7, 8] we have extended this approach to single chain under good solvent conditions, and we have shown that for the adsorption of random copolymers a simple scaling picture works very well. The scaling picture is based on the fact that the static properties of a single adsorbed chain consisting of $N$ monomers may be understood in terms of blobs. A blob is made of $g$ monomers; hence in it one of the monomer species is on the average in excess by $g^{1 / 2}$. The blob stays in its preferred solvent as long as its total interface selectivity, $\sim g^{1 / 2} \chi k_{B} T$, counterbalances its translational free energy, $k_{B} T$. This leads to $g \chi^{2}=C$, where $C$ is model dependent numerical constant. For our lattice simulation model $C$ turns out to be larger than 10 . Usually this constant $C$ (which does not play any role in a scaling argumentation) is set to unity. It follows that the number of blobs in the chain, $N / g$, equals $N \chi^{2} / C$. In fact $N \chi^{2}$ turns out to be the scaling variable of the problem, as we have confirmed through Monte Carlo simulations [7, 8]. In Ref. [9] we extended our analysis to asymmetric interface potentials, so that the chain as a whole prefers one solvent side. This problem leads to two new critical exponents (as predicted from scaling); we succeeded in evaluating these exponents based on our 
simulation data [9].

The present article is devoted to copolymer systems consisting of many chains, so that the polymer bulk density $\rho_{b}$ is significant. As we proceed to show, due to their interaction with the interface and depending on $\rho_{b}$ the copolymers display (at least) two distinct regimes. At very low $\rho_{b}$ the interface is only partially covered with adsorbed blobs; the shape of the adsorbed chains is rather flat, since their extension is larger parallel to the interface than perpendicular to it. In fact, the extension of isolated chains perpendicular to the interface does not depend on $N$, but only on the interface selectivity $\chi\left[7\right.$, 8]. As $\rho_{b}$ increases, the adsorbed, flat chains influence each other through excluded volume interactions. The chains at the interface form thus a two-dimensional semi-dilute solution. Increasing $\rho_{b}$ further leads to an interface completely saturated with blobs; thus the interface density $\rho_{s}$ depends in a complex manner on $\rho_{b}$. If the adsorption is strong enough, i.e. if the free energy of adsorption per chain is much larger than $k T$, the surface can become saturated for values of $\rho_{b}$ for which the volume phase is still highly diluted. Then the adsorbed chains form large loops, resulting in an adsorption layer width which is of the order of the radius of gyration of the polymers in the bulk. This effect is well-known for homopolymer adsorption [14, 15]. However, increasing $\rho_{b}$ beyond the saturation value a different behavior emerges: The chains extend in the direction perpendicular to the interface, since the loops of the adsorbed chains begin to stretch in a hairpin-like fashion. Such a brush-like regime for multiblockcopolymers at selective interfaces was recently predicted by Leclerc and Daoud [16]. The reason for this behavior is that the majority blobs (or blocks in Ref.[16]) can be squeezed without much loss of interface energy, since only the alternation of the two blob types across the interface fixes the chain. In this way more polymer chains 
can be adsorbed at the interface.

Our simulation results confirm this picture qualitatively. Distinct from the behavior of adsorbed homopolymers, we report here for $\rho_{b}$ larger than a characteristic value $\rho_{b}^{*}$ the stretching of the adsorbed copolymers in the direction perpendicular to the interface and their contraction parallel to it.

\section{Simulation algorithm}

Our Monte Carlo simulations for copolymers were performed using the bondfluctuation method (BFM) [10, 11]. The BFM is a lattice algorithm where each monomer is represented by a lattice cell. Thus on a three-dimensional simple cubic lattice each monomer occupies eight neighboring lattice sites. The length of a bond connecting two neighboring monomers fluctuates between 2 and $\sqrt{10}$ lattice spacings 10, 11]. Self-avoidance (the excluded volume interaction) is satisfied by not allowing any two monomers to have a lattice site in common. To avoid bond-crossing the allowed bonds are restricted to a set of 108 vectors [10, 11, 12]. Here we study the behavior of $n$ random copolymers, each of length $N$, all placed in a box of size $L \times L \times H$, with periodic boundary conditions in the $\mathrm{x}-$ and $\mathrm{y}$-directions and two impenetrable surfaces at $z=0$ and $z=H$. The copolymers are random; for each of them separately the $N$-monomer sequence consists of randomly chosen $A$ - and $B$-monomers. For adsorption we assume a symmetrical situation: the interaction energy of each monomer with its unfavorable solvent is $\chi k_{B} T$ and with its favorable solvent is zero. We let the solvent below the interface $(z \leq H / 2)$ favor A-type, and the solvent above the interface $(z \geq H / 2+1)$ B-type monomers. Note that the interface is thus at $z_{0}=(H+1) / 2$. In the Monte Carlo algorithm the chains move by position changes of their monomers, which attempt nearest neighbor steps 
on the underlying cubic lattice. A move is taken into consideration only if it satisfies the requirements of self-avoidance and of non-breaking of bonds. Furthermore, energetically unfavorable moves are statistically permitted according to the usual prescription involving the Boltzmann factor.

\section{Simulation Results}

We study the density dependence of the adsorption properties by changing $n$, the number of polymers in the $L \times L \times H$ box. Here we take $L=50$ and $H=100$ and focus on the results obtained using copolymers of length $N=64$ with a monomersolvent interaction parameter of $\chi=3.15$. Results for other copolymer lengths and for other $\chi$ parameters will be mentioned when appropriate. Note that in the single chain case $(n=1)$ the parameters $N=64$ and $\chi=3.15$ let the system be located in the well adsorbed scaling regime, see Ref.[07, 8].

An initial configuration is generated by randomly placing the first monomer of each polymer in the system and then randomly adding the subsequent monomers, such that self-avoidance and the restrictions on the bonds are obeyed. The energetic aspects of the interaction with the solvents are then taken care of by the usual Boltzmann factor; the monomer-monomer interaction is only accounted for through the excluded volume aspect. This means that both solvents are good for both species. We established numerically that the relaxation time (determined using the autocorrelation function of the radius of gyration $R_{g}$ and of its $\mathrm{z}$-component $R_{g \perp}[13$ ) is around 50,000 Monte Carlo steps (MCS), where a MCS consists of $n N$ move attempts; we thus view the copolymers as having reached equilibrium after 200,000 MCS. Averages are then calculated from the configurations obtained in the subsequent 200,000 MCS. In order to improve the statistics we average over results 
from 8 independent runs.

In Figure 1(a) we plot the densities of the A-type monomers $\left(\rho_{A}\right)$ and of the B-type monomers $\left(\rho_{B}\right)$ as a function of the height $z$. We pause to make clear how these densities are normalized. In the lattice, bond-fluctuation model used here, the maximal number of monomers which can be accommodated in the given volume is $\frac{1}{8} L^{2} H$, since due to the excluded volume restrictions each monomer blocks 8 lattice sites. In our system containing $n$ chains of $N$ monomers each the total number of monomers is $n N$; hence the average density is $\bar{\rho}=8 N n /\left(L^{2} H\right)$. Now the densities $\rho_{A}$ and $\rho_{B}$ satisfy the relation

$$
\frac{1}{H} \sum_{z}\left(\rho_{A}(z)+\rho_{B}(z)\right)=\bar{\rho}
$$

The number of polymers in Fig. 1 (a) is $n=50$. Comparing with the results for single chain, Fig.1 of Ref. [7], we find that now the monomer densities are still nonzero quite far away from the interface (i.e. from $z=10$ to $z=30$ and from $z=70$ to $z=90$ in Fig. 1(a)). Furthermore, at such distances $\rho_{A}$ and $\rho_{B}$ are equal and are independent of $z$. In this range we identify this constant with the bulk density. When approaching the impenetrable boundaries at $z=0$ and at $z=100, \rho_{A}$ and $\rho_{B}$ drop to zero. In the following we will not consider the range from $z=0$ to $z=10$ and from $z=90$ to $z=100$ any further. Close to the interface $\rho_{A}$ and $\rho_{B}$ peak on their favorable side. The densities decay smoothly on their favorable side and sharply across the selective interface, so that their values on the unfavorable side near the interface lie below the bulk density. This differs from our findings for single chain, for which we found that the bulk density is zero and that densities close to the interface on the unfavorable side display a secondary peak [0]. In Fig. 1(b) we show $\rho_{A}+\rho_{B}$ as a function of $z$. The result is a symmetric peak centered at the interface and superimposed on a bulk density background. 
In Fig. 2(a) we plot the bulk density $\rho_{b}$ against the average density $\bar{\rho}$, as given by Eq.(1). We see that $\rho_{b}$ becomes extremely low for large $\chi$ and small $\bar{\rho}$ (but does not disappear completely). The density $\rho_{b}$ increases linearly with $\bar{\rho}$ for larger $\bar{\rho}$. Fig.2(b) shows the density at the interface $\rho_{s}=\left(\rho_{A}(H / 2)+\rho_{B}(H / 2)+\rho_{A}(H / 2+\right.$ 1) $\left.+\rho_{B}(H / 2+1)\right) / 2$ as a function of $\bar{\rho}$. For small $\bar{\rho}, \rho_{s}$ increases rapidly, while for larger $\bar{\rho}$ it increases more slowly, the cross-over region being around $\bar{\rho} \simeq 0.02$. From the Figure the almost linear increase of $\rho_{s}$ in the range of large $\bar{\rho}$ is also clear. Fig. 2(c) shows a plot of $\rho_{s}$ versus $\rho_{b}$, where again the two regimes can be seen. To understand these regimes we plot in Fig. 2(d) $\rho_{s}-\rho_{b}$ against $\bar{\rho}$. In terms of Fig.1(b) $\rho_{s}-\rho_{b}$ is in fact the peak's height relative to the background. We see from Fig. 2(d) that $\rho_{s}-\rho_{b}$ attains its maximum around $\bar{\rho}_{c} \simeq 0.02$. For $\bar{\rho}<\bar{\rho}_{c}, \rho_{s}-\rho_{b}$ increases with increasing $\bar{\rho}$ while for $\bar{\rho}>\bar{\rho}_{c}$ it decreases with increasing $\bar{\rho}$. This means that in the low $\bar{\rho}$ regime $\left(\bar{\rho}<\bar{\rho}_{c}\right)$ adding more polymers to the system leads mainly to an increase in density at the interface, whereas for higher $\bar{\rho}$ values $\left(\bar{\rho}>\bar{\rho}_{c}\right)$ adding more polymers to the system leads to an overall increase in density in the bulk. In the second regime both $\rho_{s}$ and $\rho_{b}$ increase almost linearly with $\bar{\rho}$, whereas $\rho_{s}-\rho_{b}$ decreases roughly linearly with increasing $\bar{\rho}$. Extrapolating the linear dependence in the second regime of Fig. $2(\mathrm{~d})$ to $\rho_{s}-\rho_{b}=0$ leads to $\bar{\rho} \simeq 0.95$. We note that a third regime may exist at still higher $\bar{\rho}$ values, when the copolymer concentration in the bulk reaches the semi-dilute range, but investigations in exploring rather dense systems are beyond the scope of the present paper.

For the record, we like to point out that varying the copolymers' length, using $N=64$ and $N=128$, we obtained plots almost identical to those shown in Fig.2. For different $\chi$ parameters $(\chi=2.20,3.15$ and 4.20$)$, we find that $\rho_{b}$ is independent of $\chi$ while $\rho_{s}$ increases with increasing $\chi$. 
The difference between $\rho_{A}$ and $\rho_{B}$ provides a means to quantify the interfacial selectivity. Fig. 3(a) displays the data for $n=50$. We plot $\rho_{A-B} \equiv \rho_{A}-\rho_{B}$ for $z \leq H / 2$ and $\rho_{A-B} \equiv \rho_{B}-\rho_{A}$ for $z \geq H / 2+1$; this leads to a symmetric peak. We find in this density regime $(\bar{\rho} \simeq 0.1)$ that the width of the peak is independent of $N$ but is controlled by $\chi$. Fig. 3(b) shows how the width of the peak in Fig. 3(a) varies as a function of $\bar{\rho}$ for different $\chi$. Here the width is taken as full width at half-height (FWHH), i.e. the difference $z_{2}-z_{1}$, with $\rho_{A-B}\left(z_{1}\right)=\rho_{A-B}\left(z_{2}\right)=\frac{1}{2} \rho_{A-B}\left(z_{0}\right)$, where $\rho_{A-B}\left(z_{0}\right)$ is the density at the interface (the peak's height in Fig. 3(a)). Since our model is based on a lattice, we had to interpolate between the discrete values, in order to obtain the (non-integer) FWHH. We have also evaluated numerically the second moment of the distribution of Fig. 3(a) and found indications that the second moment may diverge. In Fig. 3(c) we show the peak's height $\rho_{A-B}$ as a function of $\bar{\rho}$ and we find that the relationship is linear above $\bar{\rho}_{c}$.

Keeping in mind that the interfacial selectivity affects mostly the chains close to the interface, i.e. the adsorption layer, see Fig. 3(b), we turn now to a comparative study of the polymers' behavior around the interface and also away from it. For this we compute $R_{g \perp}$ and $R_{g \|}$, the $\mathrm{z}^{-}$and the xy-components of the radius of gyration of each copolymer. The center of mass of the $k$ th polymer is $\vec{R}_{C M}^{(k)}=$ $\sum_{i=1}^{N} \vec{R}_{i}^{(k)} / N$, where $\vec{R}_{i}^{(k)}=\left(x_{i}^{(k)}, y_{i}^{(k)}, z_{i}^{(k)}\right)$ are the coordinates of the $i$ th monomer within the $k$ th chain. For the $k$ th chain, $R_{g \perp}^{(k)}=\left(\sum_{i=1}^{N}\left(z_{i}^{(k)}-z_{C M}^{(k)}\right)^{2} / N\right)^{1 / 2}$, and $R_{g \|}^{(k)}=\left(\frac{1}{2} \sum_{i=1}^{N}\left[\left(x_{i}^{(k)}-x_{C M}^{(k)}\right)^{2}+\left(y_{i}^{(k)}-y_{C M}^{(k)}\right)^{2}\right] / N\right)^{1 / 2}$. Now we have to specify which copolymers belong to the space around the interface. For this we use a $\sigma$-criterion: If the z-component of the polymer's center of mass is within a distance $\sigma$ from the interface, i.e. if $\left|z_{C M}^{(k)}-z_{0}\right| \leq \sigma$, we view the polymer as being near the interface, otherwise as being far from it (here again we disregard the far-off regions close to the 
fixed boundaries). Now, evidently $2 \sigma$ should be taken larger than the peak's FWHH. Since the FWHH, Fig. 3(b), depends on $\bar{\rho}$, we choose $\sigma=10$ in what follows (we checked that the choice of $\sigma$ does not change the features reported below, provided that $2 \sigma$ is reasonably larger than the FWHH). We now average $R_{g \perp}^{(k)}$ and $R_{g \|}^{(k)}$ for the polymers near the interface to obtain $R_{g \perp}$ and $R_{g \|}$; we do the same for the polymers far from the interface, which leads to $\widetilde{R}_{g \perp}$ and $\widetilde{R}_{g \|}$. In Fig. 4(a) and Fig. 4(b) we present plots of $R_{g \perp}$ and $R_{g \|}$ and of $\widetilde{R}_{g \perp}$ and $\widetilde{R}_{g \|}$ as functions of $\bar{\rho}$.

Let us first consider the bulk phase, away from the interface. As can be readily inferred from Fig. 4(b), $\widetilde{R}_{g \perp}$ is close to $\widetilde{R}_{g \|}$ for all $\bar{\rho}$, which means that the bulk copolymers' shape is not affected by the interface and that it is isotropic in space. Note that the large fluctuations of the data points for small $\bar{\rho}$ values arise from the fact that almost no chains are located in the bulk until the interface is saturated, see also Fig. 2(a).

The situation is completely different for polymers near the interface, Fig. 4(a). For very small values of $\bar{\rho}, R_{g \perp}$ is considerably smaller than $R_{g \|}$; hence adsorbed chains tend to be rather flat. Fig.4(a) shows that $R_{g \|}$ decreases monotonically with increasing $\bar{\rho}$, whereas the opposite is true for $R_{g \perp}$. This is analogous to the behavior of adsorbed homopolymers, as discussed by Bouchaud and Daoud [14. Comparing Fig.4(a) with Fig.2(c) one notes that the chain's extension is, in contrast to the surface density, a smooth function of $\bar{\rho}$ also at the saturation value $\bar{\rho}_{c}$. However, increasing the density beyond the value $\bar{\rho}^{*} \simeq 0.05$, where $R_{g \|}=R_{g \perp}$, the extension perpendicular to the interface gets to be larger than the parallel extension. This is in accordance with Ref. [16], which predicts (as discussed in the Introduction) that above a characteristic bulk concentration the loops of the adsorbed chains will stretch in the direction perpendicular to the interface, in a hairpin-like fashion. 
Note that also the chain's extension parallel to the interface, $R_{g \|}$, decreases below its bulk value $\widetilde{R}_{g \|}$ (compare Figs.4(a) and (b)), i.e. the adsorbed chains begin to get squeezed parallel to the interface. Thus varying the bulk concentration only by a small amount $\left(0<\rho_{b}<0.1\right)$ the chain's geometry changes from a flat, pancake-like shape into a brush-like assembly of stretched loops. This picture is also supported by the behavior of $\rho_{s}$ as a function of $\bar{\rho}$ in the saturated surface regime $\left(\bar{\rho}>\bar{\rho}_{c}\right)$. As can be inferred from Fig.2b, the surface concentration increases even beyond the saturation threshold $\bar{\rho}_{c}$ (which is given in Figs.2 already by the second numerical point).

\section{Conclusions}

We have investigated the behavior of many random copolymers in the presence of a selective interface. Previous studies [3, 6, 7, 8] showed that a simple scaling picture works very well for random copolymer adsorption. In this paper we have extended our previous work to consider the effects of sizeable chain concentrations. We found that there are at least two regimes controlled by the polymer density, as can be inferred from Figs.2 and Fig.4a.

In the very low density regime (except for the adsorption mechanism) copolymers at interfaces behave similarly to homopolymers at surfaces. Starting from a single chain, which in the adsorbed state is flat, an increase in the chains' density leads to their crowding at the interface. As usual for polymer adsorption, the interface may be fully covered by chains even when the bulk density is still highly diluted (compare the surface density $\rho_{s}$ and the bulk density $\rho_{b}$ for the second numerical point in Fig. 2(c)). This can be easily understood from the fact that the adsorption energy per chain is in most cases a huge quantity compared to the translational free energy per chain, i.e. to $k T$. 
In the second regime, however, where the interface is already covered, the only way of adding more chains to it is to squeeze the chains in a brush-like fashion. As a consequence, $R_{g \perp}$, the radius of gyration of the adsorbed chains perpendicular to the interface exceeds the average value $\widetilde{R}_{g \perp}$ in the bulk. A means of picturing this situation is a brush-like assembly of stretched loops [16].

\section{Acknowledgments}

We are grateful to M. Daoud and T. Ohta for useful discussions. This work was supported by the Deutsche Forschungsgemeinschaft, by the Fonds der Chemischen Industrie, by PROCOPE administrated by the DAAD, and by the Monbusho Grantin-Aid, Japan. G. P. thanks both the Alexander von Humboldt Foundation and the Japan Society for the Promotion of Science for support during different stages of the work. 


\section{References}

[1] H. R. Brown, V. R. Deline and P. F. Green, Nature (London) 341, 221 (1989).

[2] C.-A. Dai, B.J. Dair, K. H. Dai, C. K. Ober, E. J. Kramer, C.-Y. Hui and L. W. Jelinski, Phys.Rev.Lett. 73, 2472 (1994).

[3] T. Garel, D. A. Huse, S. Leibler and H. Orland, Europhys.Lett. 8, 9 (1989).

[4] C. Yeung, A. C. Balazs and D. Jasnow, Macromolecules 25, 1357 (1992).

[5] A. Grosberg, S. Izrailev and S. Nechaev, Phys.Rev. E 50, 1912 (1994).

[6] J. -U. Sommer and M. Daoud, Europhys. Lett. 32, 407 (1995).

[7] G. Peng, J.-U. Sommer and A. Blumen, Phys. Rev. E 53, 5509 (1996)

[8] J.-U. Sommer, G. Peng and A. Blumen, J. Phys. II France 6, 1061 (1996).

[9] J.-U. Sommer, G. Peng and A. Blumen, J. Chem. Phys. 105, 8376 (1996)

[10] I. Carmesin and K. Kremer, Macromolecules 21, 2819 (1988).

[11] H. P. Deutsch and K. Binder, J. Chem. Phys. 94, 2294 (1991).

[12] H.-L. Trautenberg, Th. Hölzl and D. Göritz, Comput. Theoret. Polym. Sci. 6, $135(1996)$

[13] P.-Y Lai and K. Binder, J. Chem. Phys. 95, 9288 (1991).

[14] E. Bouchaud and M. Daoud J.Physique 48, 1919 (1987)

[15] G.J. Fleer, M.A. Cohen Stuart, J.M.H.M. Scheutjens, T. Cosgrove and B. Vincent, Polymers at Interfaces, Chapman \& Hall, London (1993)

[16] E. Leclerc and M. Daoud Macromolecules 30, 293 (1997) 


\section{Figure Captions}

Figure 1(a): Densities of the A-monomers $\left(\rho_{A}\right)$ (diamonds) and of the B-monomers $\left(\rho_{B}\right)$ (crosses) at the height $z$. Here the number of polymers is $n=50$, the length of all polymers is $N=64$, and the monomer-solvent interaction parameter is $\chi=3.15$.

Figure 1(b): Display of $\left|\rho_{A}+\rho_{B}\right|$ vs. $z$, see Fig.1(a) for definitions.

Figure 2(a): The bulk density $\left(\rho_{b}\right)$ vs. the average density $(\bar{\rho})$, see text for details.

Figure 2(b): The density at the interface $\left(\rho_{s}\right)$ vs. $\bar{\rho}$.

Figure 2(c): $\rho_{s}$ vs. $\rho_{b}$.

Figure 2(d): $\rho_{s}-\rho_{b}$ vs. $\bar{\rho}$.

Figure 3(a): $\rho_{A-B}$ (the absolute value of $\rho_{A}-\rho_{B}$ ) vs. the height $z$. Here $n=50$.

Figure 3(b): The FWHH of Fig.3(a) vs. $\bar{\rho}$ for three different $\chi$ parameters, $\chi=2.20$ (diamonds), $\chi=3.15$ (crosses), $\chi=4.20$ (squares).

Figure 3(c): $\rho_{A-B}\left(z_{0}\right)$ vs. $\bar{\rho}$, with $z_{0}$ being the interface's location.

Figure 4(a): Radius of gyration for polymers near the interface: $R_{g \perp}$ (diamonds) and $R_{g \|}$ (crosses) vs. $\bar{\rho}$. 
Figure 4(b): Radius of gyration for polymers away from the interface: $\widetilde{R}_{g \perp}$ (diamonds) and $\widetilde{R}_{g \|}$ (crosses) vs. $\bar{\rho}$. 


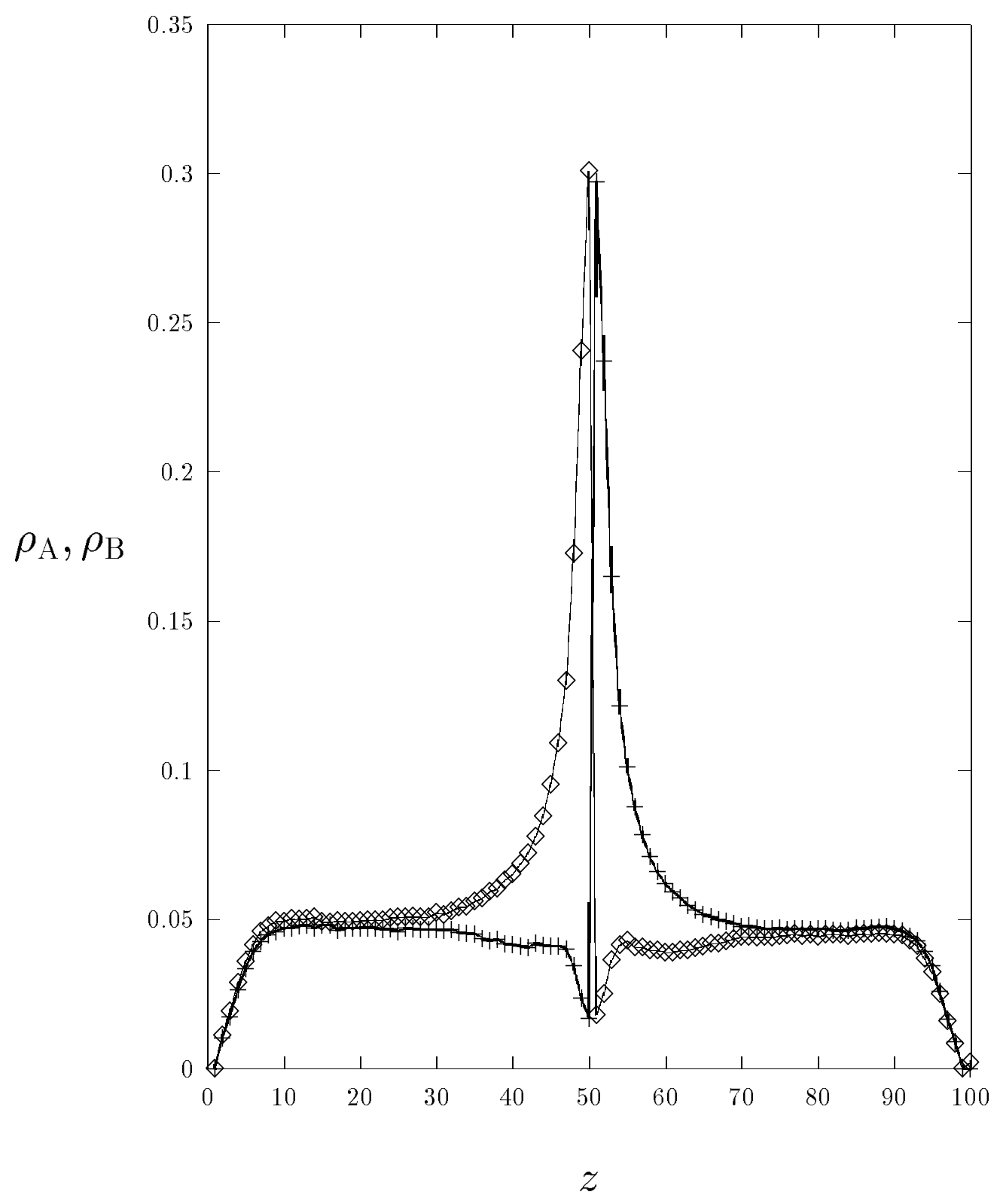

Fig. 1a 


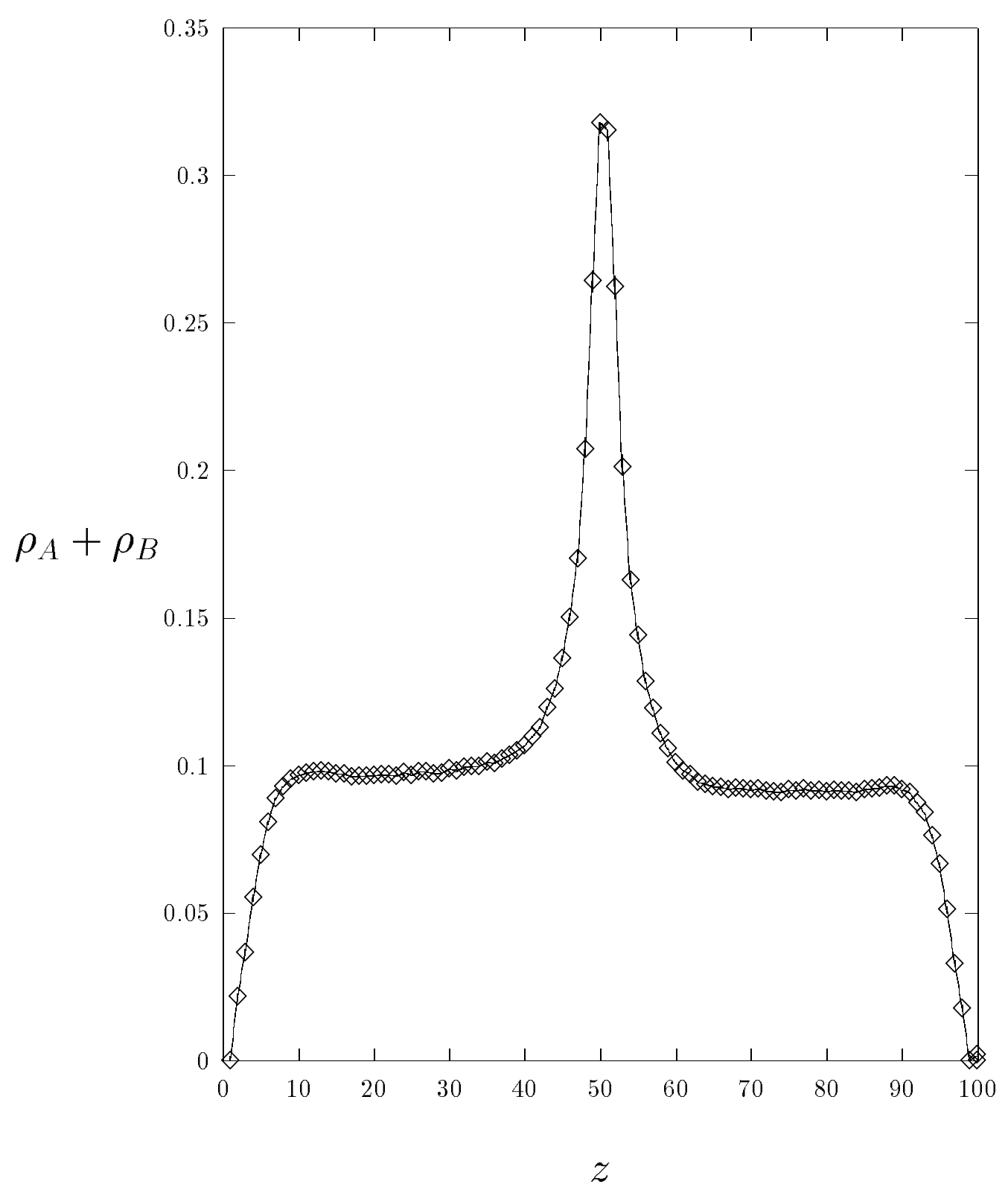

Fig. 1b 


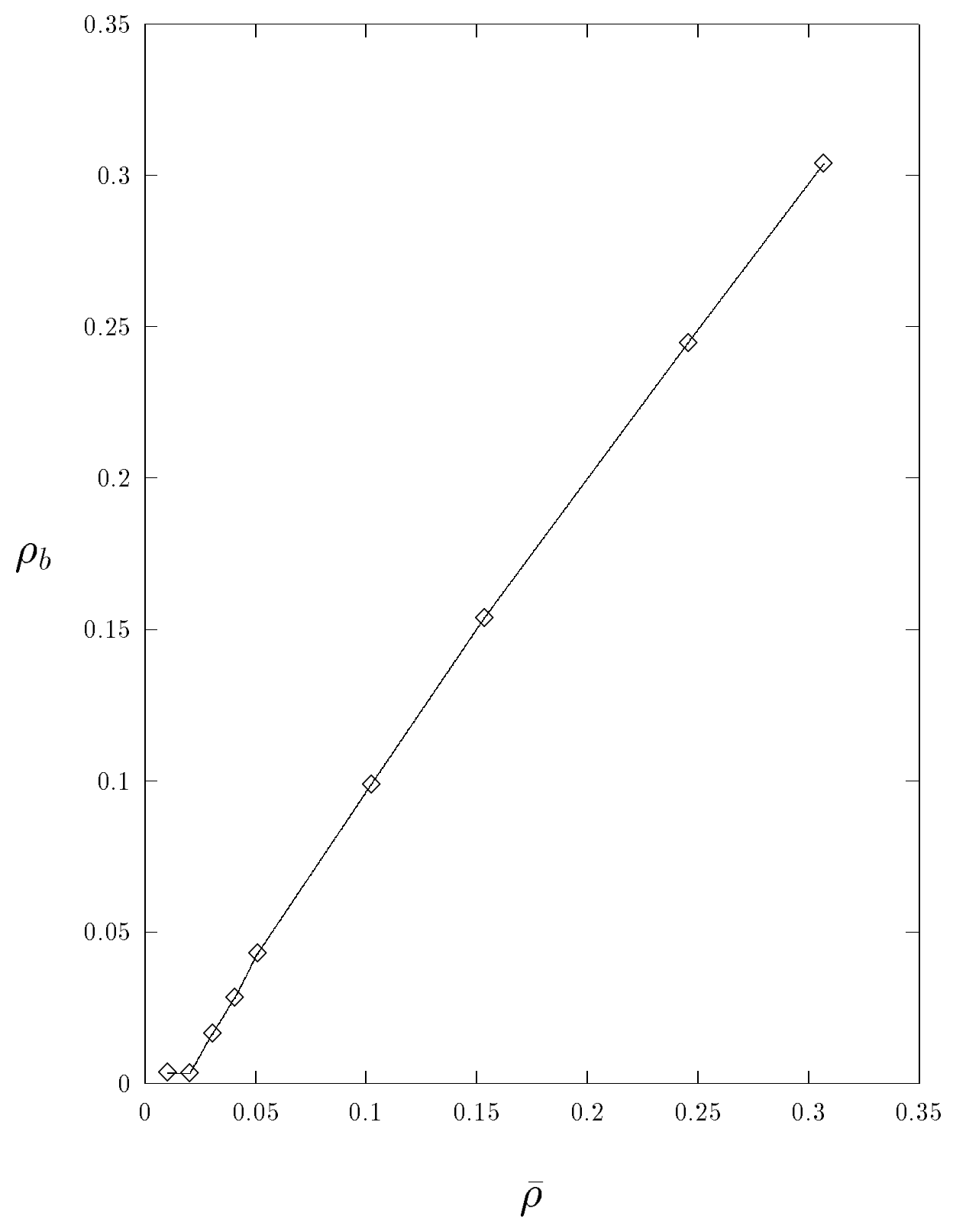

Fig. 2a 


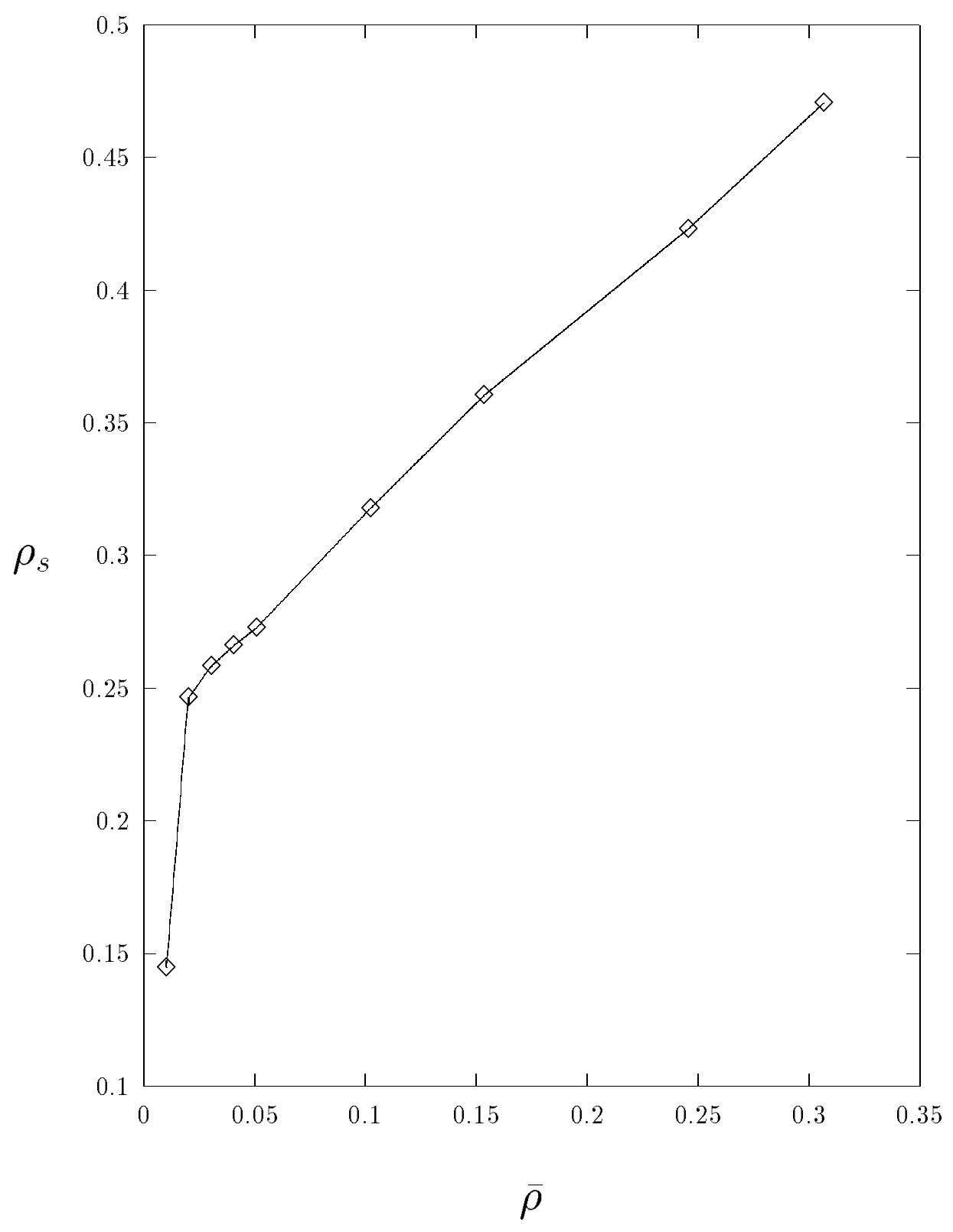

Fig. 2b 


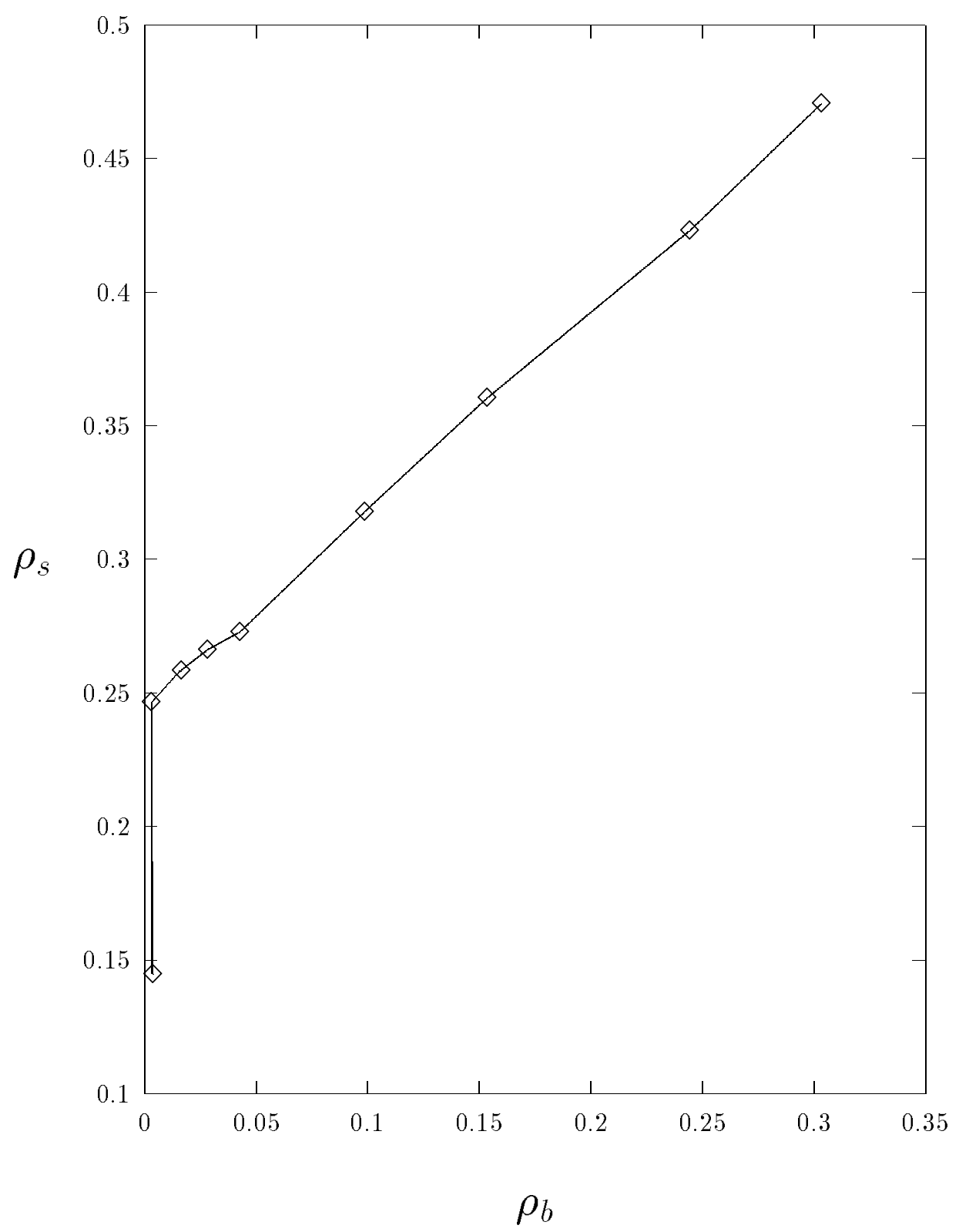

Fig. 2c 


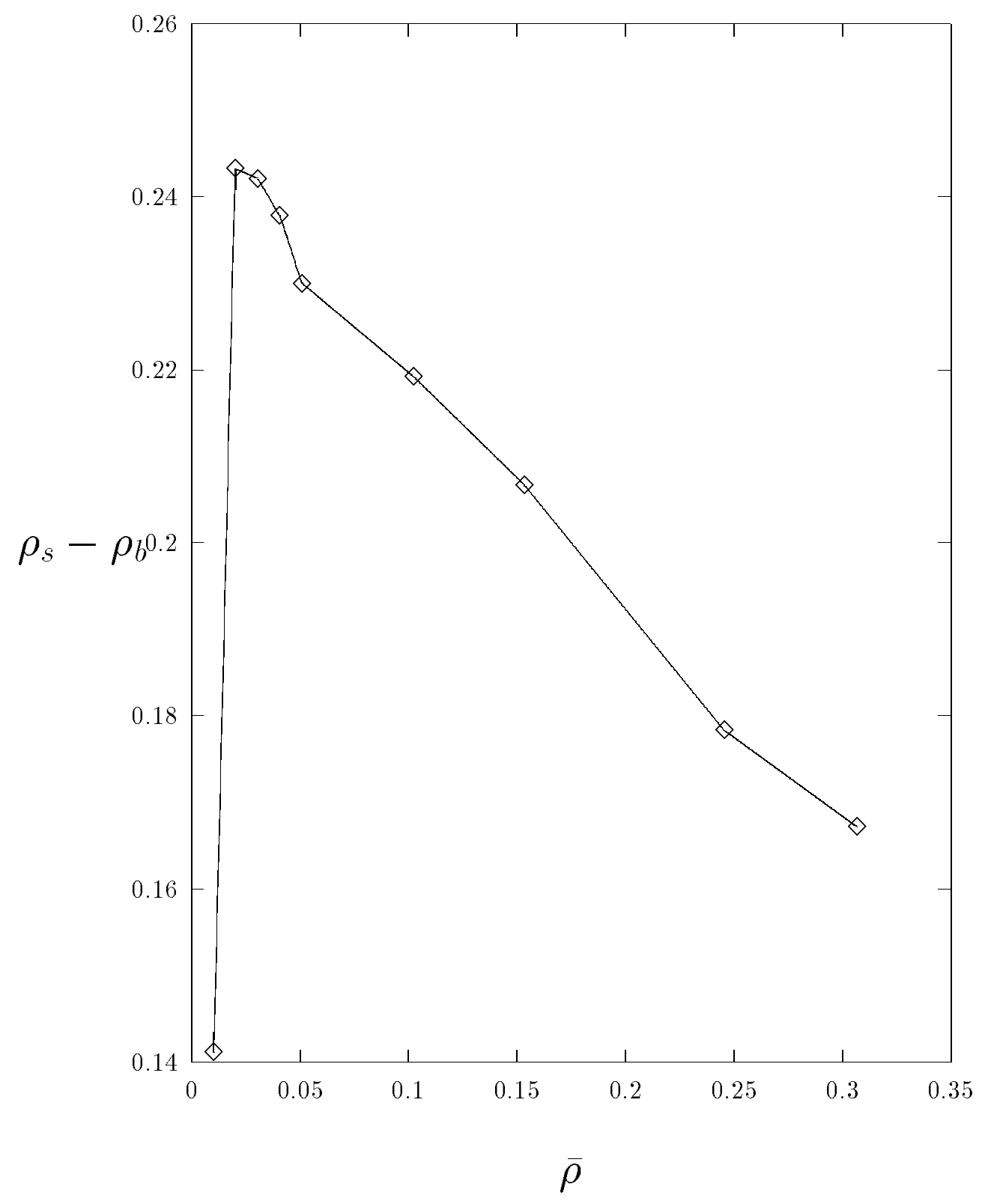

Fig. 2d 


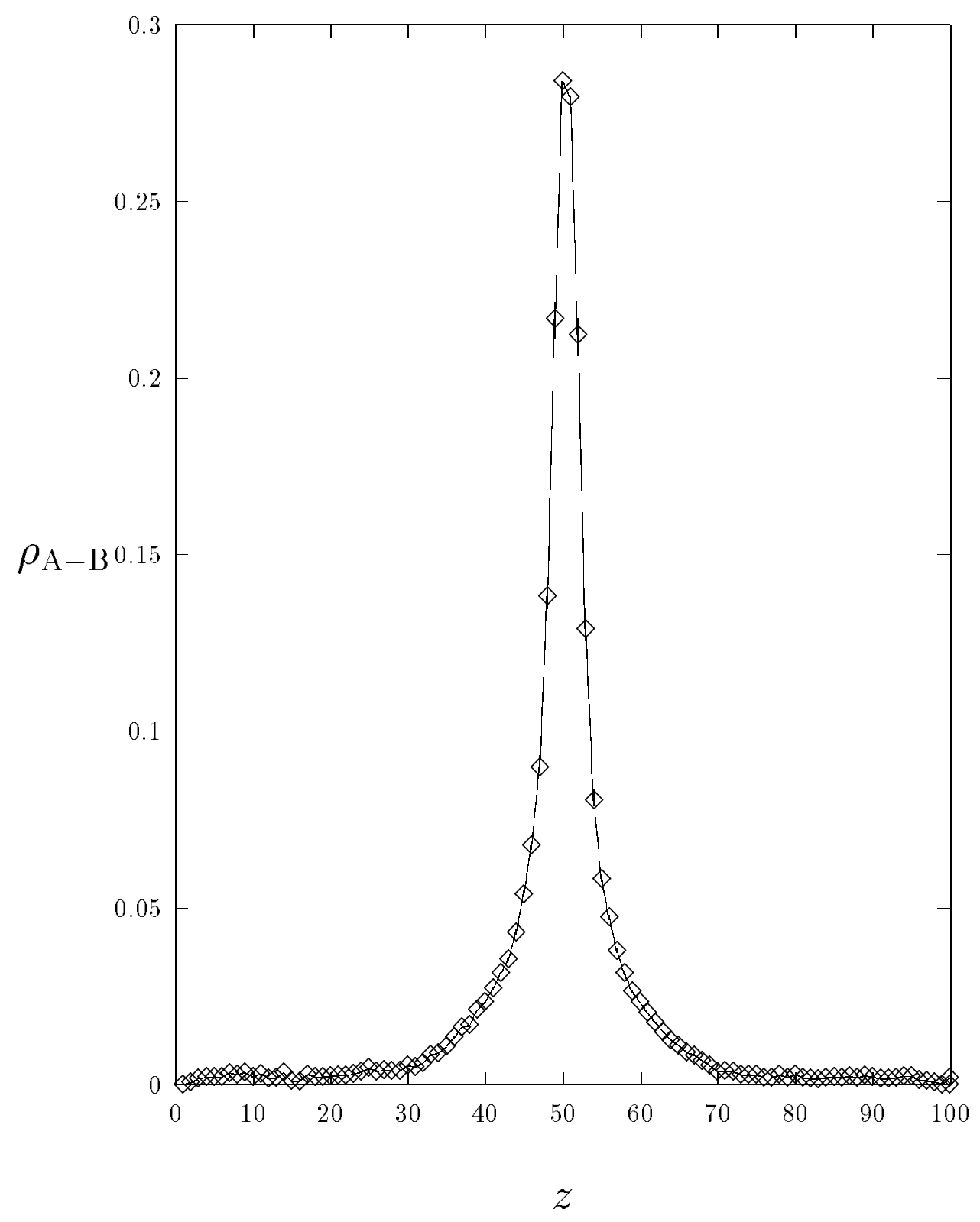

Fig. 3a 


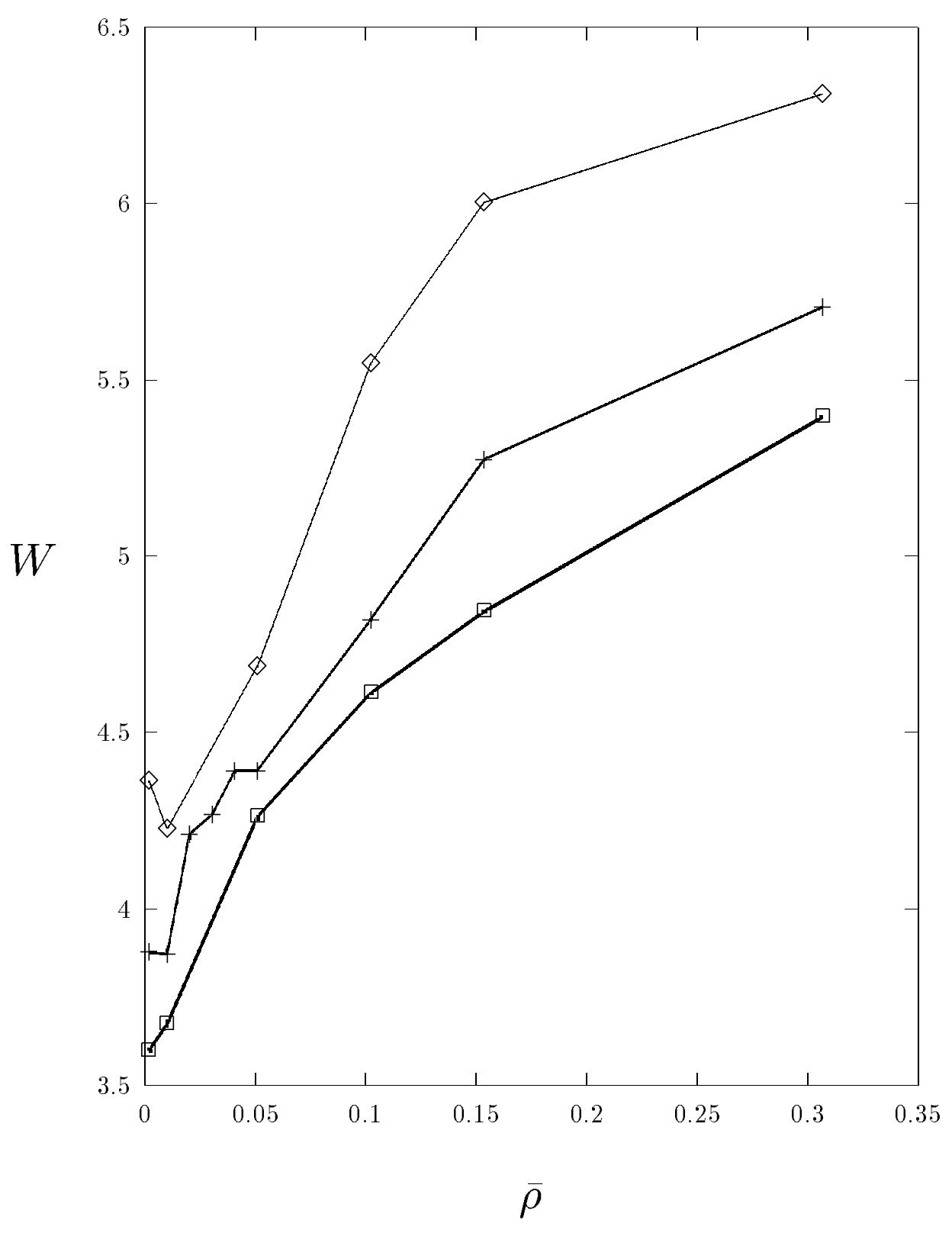

Fig. 3b 


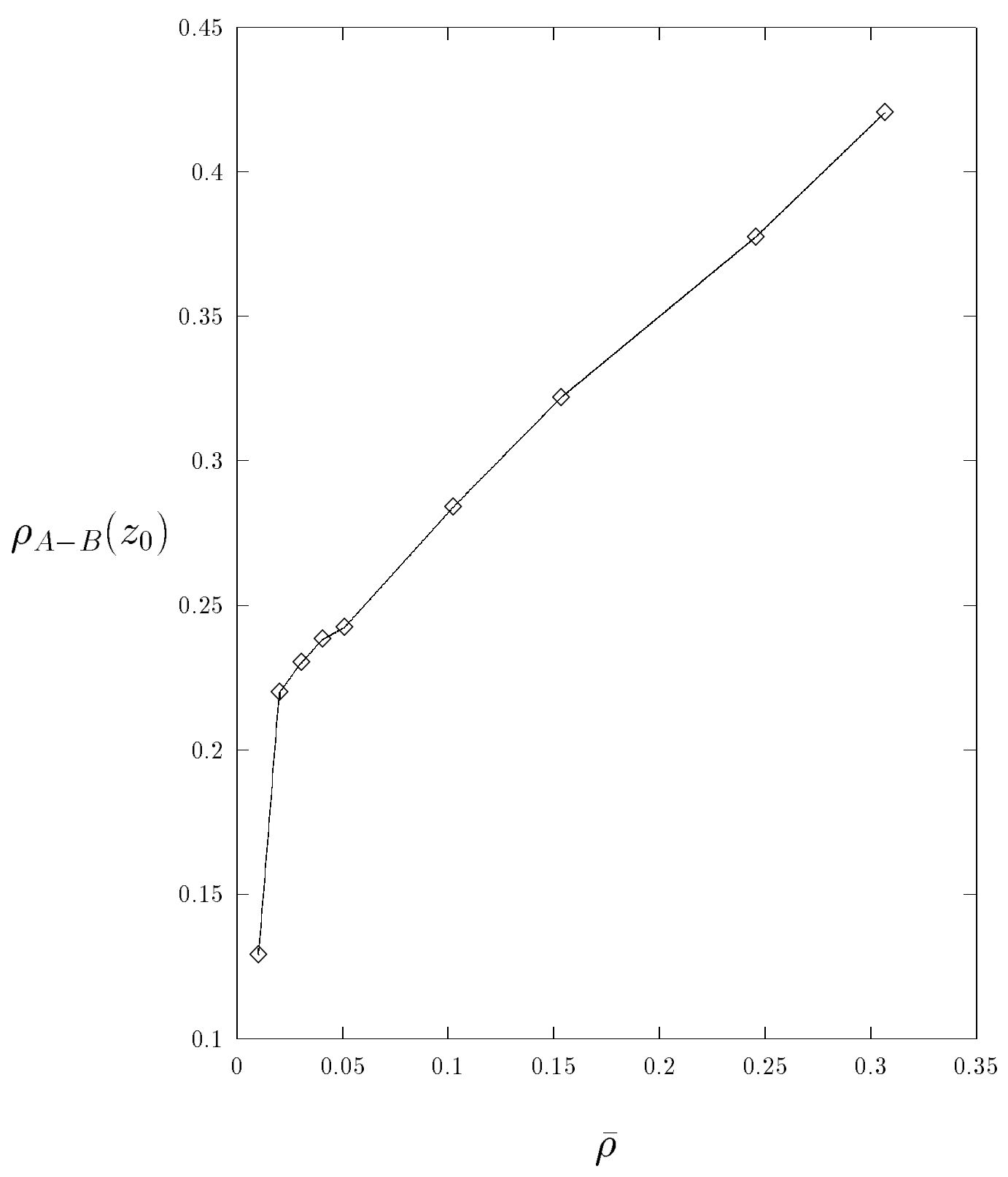

Fig. 3c 


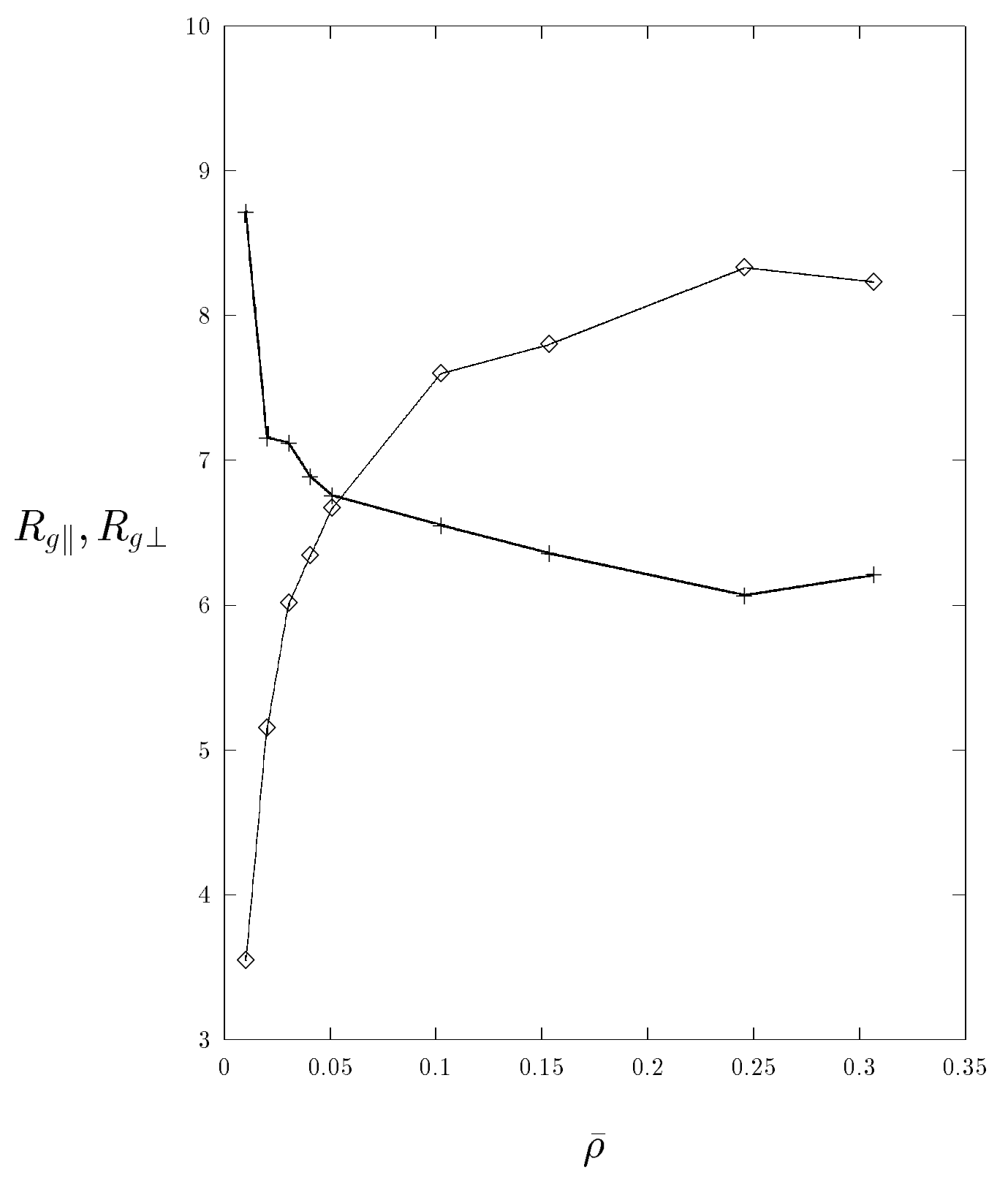

Fig. 4a 


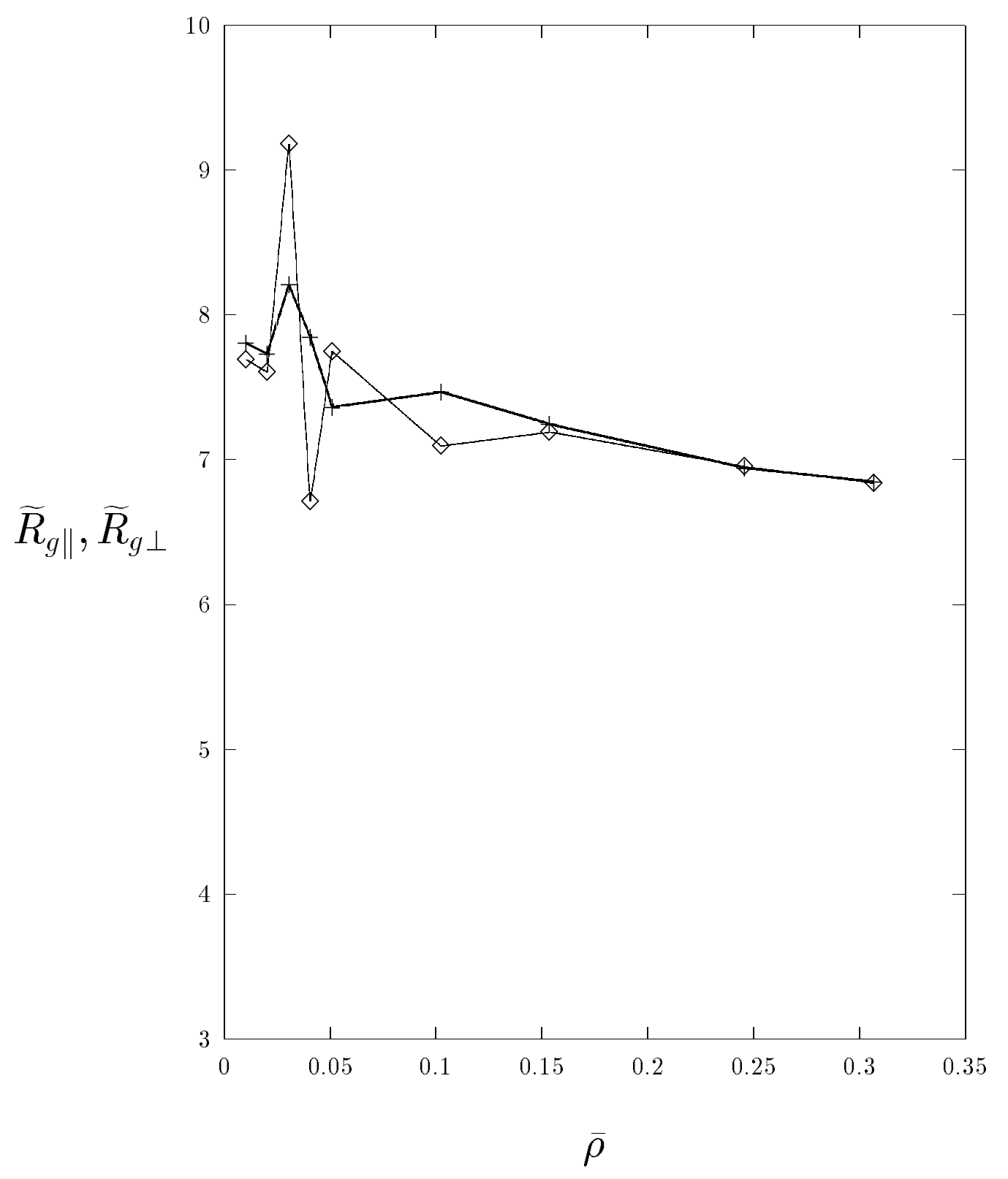

Fig. 4b 